\title{
Changes in anterior chamber flare and cells following cataract surgery
}

\author{
Sanjay M Shah, David J Spalton
}

\begin{abstract}
The laser flare cell meter allows rapid noninvasive quantification of aqueous flare and cells. In this prospective study laser photometry was used to document the recovery of the blood-aqueous barrier in 27 normal eyes following cataract surgery. Aqueous flare and cells were highest on the first postoperative day, declining rapidly in the first week and returning to preoperative levels by 3 months. In six eyes $(22 \cdot 2 \%)$ there was an increase in either flare and cells or flare alone during the first postoperative week which was associated with a delayed recovery of the blood-aqueous barrier for up to 1 month following surgery. A consensual flare response was found to occur in the fellow eye in five patients $(18 \cdot 5 \%)$. (Brf Ophthalmol 1994; 78: 91-94)
\end{abstract}

The laser flare cell meter (Kowa FC 1000) allows quantification of aqueous protein and particulate matter by computer analysis of light scattered by a helium neon beam projected into the anterior chamber. ${ }^{1}$ The technique is rapid and noninvasive and requires minimal patient cooperation. Flare measurements have been shown to be accurate, to have a high reproducibility both in vitro and in vivo (8-12\%), ${ }^{12}$ and to correlate well with clinical grading of flare $^{3}$ as well as with anterior chamber protein levels in the normal eye and in eyes with mild damage to the bloodaqueous barrier (BAB). ${ }^{4}$ The technique is more sensitive and accurate than anterior chamber fluorophotometry in the assessment of BAB function.

There is little published information available on the changes in aqueous flare and cells in the normal eye in the first 6 months following cataract surgery. We documented the recovery of the $\mathrm{BAB}$ in the normal pseudophakic eye in the first 6 postoperative months. A previous study using fluorophotometry indicated that cataract surgery induced BAB changes in the fellow eye which might have clinical relevance under some circumstances. ${ }^{6}$ We therefore also investigated whether there was evidence of a consensual response to surgery in the $\mathrm{BAB}$ of the fellow eye.

Medical Eye Unit, St Thomas's Hospital, London

S M Shah

D J Spalton

Correspondence to:

D J Spalton, The Medical Eye Unit, St Thomas's Hospital Lambeth Palace Road, London SE1 7EH.

Accepted for publication 17 August 1993 multiplier tube, and a personal computer. The helium neon beam is projected into the anterior chamber and light scattered from within a sampling window (volume $0.075 \mathrm{~mm}^{3}$ ) is detected by the photomultiplier tube. This scattered light is analysed to produce an aqueous flare value expressed in photons/ms and a cell count as a number $/ 0.075 \mathrm{~mm}^{3}$. All values for flare and cells in this study are reported in these units. Each scan takes 1 second. Measurements were made with an undilated pupil (pupillary dilatation significantly affects flare ${ }^{2}$ ). To avoid the introduction of bias five sequential scans were accepted and averaged provided that the background scatter was less than $15 \%$ on each individual scan. ${ }^{12}$ Therefore the results of an individual patient are expressed as a mean (SD). Calibration was checked according to the manufacturer's instruction at frequent intervals throughout the study. The study conformed to the ethical requirements of the West Lambeth Health Authority.

\section{CATARACT SURGERY}

Twenty seven eyes of 26 patients (mean age 68.6 years, range $49-80,10$ male) were studied. All patients preoperatively had otherwise normal eyes with no other detectable ocular pathology apart from the cataract. They were all systemically well and, in particular, were not taking any topical or systemic anti-inflammatory medication. There was one diabetic patient (with no evidence of retinopathy) included in the study. All surgery was performed by a single surgeon using a standardised endocapsular surgical technique. Briefly, this consisted of an incision into the vascularised limbus, a linear capsulotomy under Healon, manual expression of the nucleus, and automated irrigation aspiration using balanced salt solution. The implant was inserted into the capsular bag under Healon and the

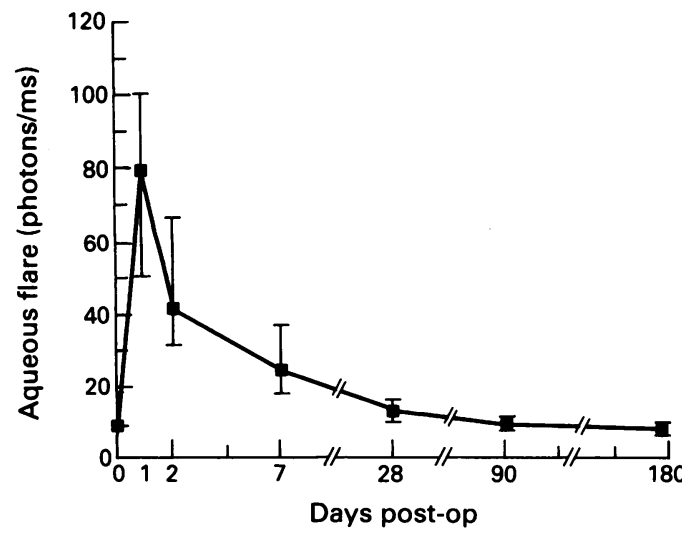
anterior chamber. ${ }^{1}$ The instrument consists of three main components: a helium neon laser slitlamp, a binocular microscope fitted with a photo-

\section{Subjects and methods}

\section{LASER FLARE CELL METER}

The Kowa laser flare cell meter (FC 1000) measures aqueous flare and cells by the light measures aqueous flare and cells by the light
Figure 1 Changes in median (with 95\% confidence interval) aqueous flare in the first 6 months following cataract surgery. 
anterior capsule excised. Healon was removed and the wound closed using $10 / 0$ prolene. Subconjunctival cefuroxime $(100 \mathrm{mg})$ was given at the end of surgery. No subconjunctival steroids were used. Following surgery all patients received the same regimen (topical dexamethasone $0 \cdot 1 \%$ and neomycin $0 \cdot 35 \%$ four times daily for 1 week reducing to twice daily for a further 3 weeks) unless otherwise clinically indicated. Laser flare and cell measurements were performed on both eyes of all patients preoperatively and on postoperative days 1,2 , and 7 and at 1,3 , and 6 months following surgery. These time points were chosen for their potential clinical relevance. The preoperative and postoperative clinical assessments were performed in all cases by the same single observer. Laser flare measurements were in most cases performed independently by a different observer.

\section{STATISTICAL ANALYSIS}

Postoperative aqueous flare values were not normally distributed even after logarithmic transformation. The recovery of the $\mathrm{BAB}$ and the consensual reaction in the fellow eye were therefore assessed by a comparison of postoperative laser flare and cell measurements with preoperative values using the Wilcoxon signed rank test. The Mann-Whitney test was used to compare flare and cell values between groups of patients at each postoperative visit. $p$ Values of $<0.05$ were considered statistically significant. All variables are presented as the median with the $95 \%$ confidence interval $(95 \% \mathrm{CI})$ unless otherwise stated. ${ }^{7}$

\section{Results}

All surgery was uneventful; $92 \cdot 6 \%$ of all eyes achieved a best corrected visual acuity at 3 months of $6 / 12$ or better. One patient developed a postoperative iritis that required a change in the standard postoperative medication regimen. The median postoperative flare and cell values are presented in Figures 1 and 2. These were highest on the first postoperative day (median aqueous flare $79 \cdot 6,95 \%$ CI 50.6-100.8, median cell count $47 \cdot 4,95 \%$ CI $37-61 \cdot 2$ ) declining rapidly in the first week with a more gradual recovery to preoperative levels by 3 months

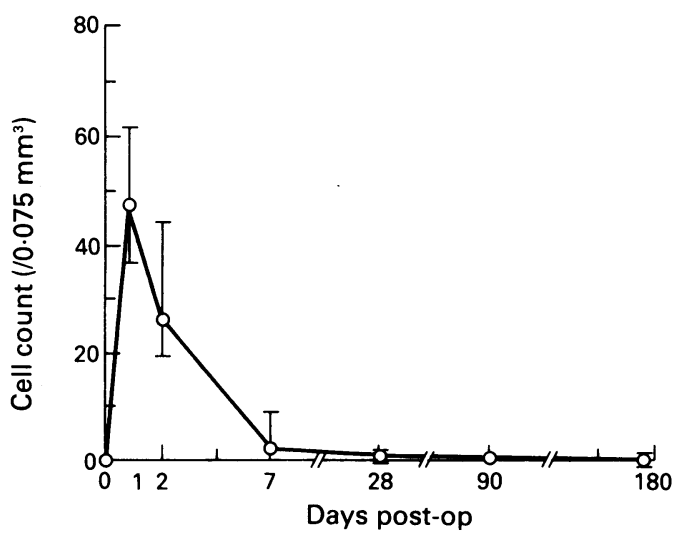

Figure 2 Changes in median (with $95 \%$ confidence interval) anterior chamber cell count in the first 6 months following cataract surgery.

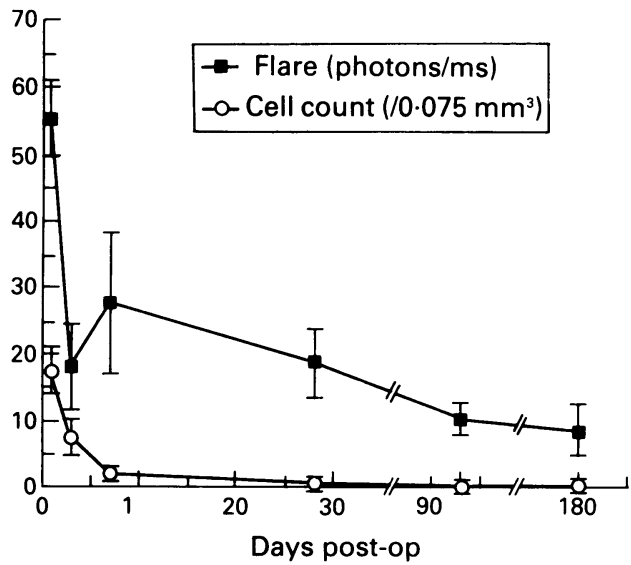

Figure 3 Aqueous flare and cells following cataract surgery in a 76-year-old woman (patient No 22). There is an asymptomatic increase in flare between day 2 and day 7 without an increase in cells. (Flare and cells, mean (SD).)

postoperatively. Median aqueous flare at 3 months $(9 \cdot 2,7.9-12.5)$ was not significantly different $(p=0 \cdot 33$, Wilcoxon signed rank) from the preoperative flare value $(9 \cdot 3,7 \cdot 8-10 \cdot 3)$. The median cell count at 3 months $(0 \cdot 2,0-0.4)$ had almost returned $(p=0 \cdot 04$, Wilcoxon signed rank) to the preoperative level $(0,0-0 \cdot 2)$.

In this group of 27 eyes it was possible to distinguish two variations from the normal pattern of postoperative recovery described above. In $21(77 \cdot 8 \%)$ patients there was an uneventful recovery (as shown in Figs 1 and 2). In these patients aqueous flare decreased by a median of $48 \cdot 2 \%(95 \%$ CI $34 \cdot 9-62 \cdot 4 \%)$ and cell count by a median of $94 \cdot 3 \%$ (95\% CI $87 \cdot 5-$ $96.4 \%$ ) between the second and seventh postoperative day. In four $(14.8 \%)$ patients, however, there was an increase in flare between day 2 and day 7 (patient No 22, Fig 3) which was asymptomatic and not clinically detectable. In these four eyes flare increased by a median of $32 \cdot 4 \%$ (range $9 \cdot 2-119 \cdot 4 \%$ ) between day 2 and day 7 , this increase in flare being significantly different $(p=0.002$, Mann-Whitney test) from the decrease in flare seen over the same interval in the eyes with an uneventful recovery. In two of these patients the flare value on day 7 even exceeded the value on the first postoperative day.

Two $(7 \cdot 4 \%)$ patients had an increase in both

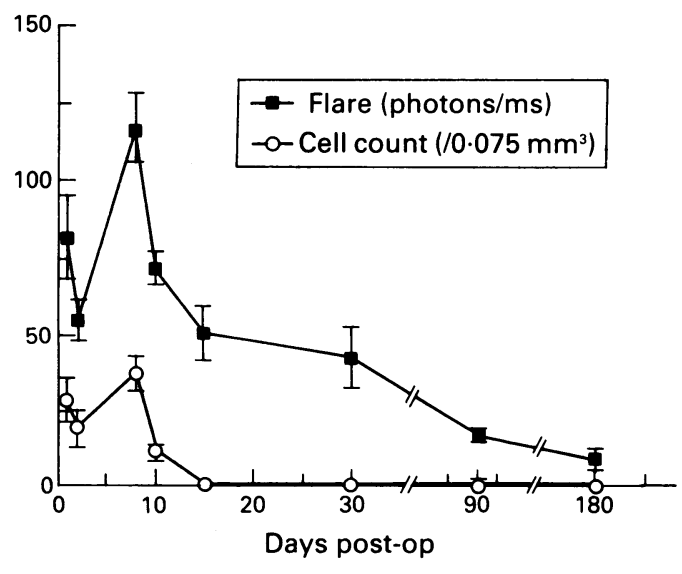

Figure 4 Aqueous flare and cells following cataract surgery in a 62-year-old man (patient No 6). There was a symptomatic increase in both flare and cells between day 2 and day 7 . (Flare and cells, mean (SD).) 
Figure 5 Aqueous flare and cells following cataract surgery in a 68-year-old man. There was a marked increase in flare between the second and seventh postoperative day in the operated eye and $a$ simultaneous consensual response in flare in the fellow eye (operated on 6 weeks previously).

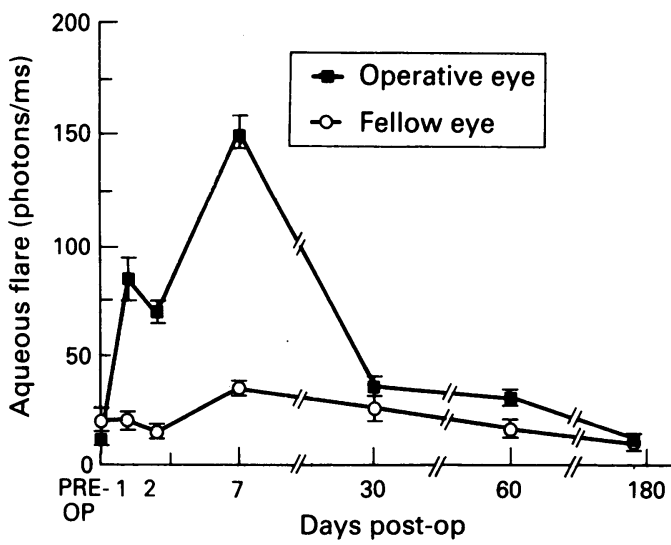

flare $(+8 \cdot 7 \%$ and $+113 \cdot 5 \%)$ and cells $(+291 \cdot 7 \%$ and $+90.9 \%$ ) between the second and seventh postoperative day (patient No 6, Fig 4). In these two patients the cell count on day 7 exceeded the cell count on the first postoperative day. This increase in flare and cells was symptomatic, with increased redness and pain clinically detectable and in one patient it required intensive topical steroid therapy (this was the only patient who required a change in topical medication in this series).

Therefore in six eyes there was an increase in aqueous flare in the first postoperative week. Median aqueous flare was still significantly higher $(\mathrm{p}=\mathbf{0} \cdot 02$, Mann-Whitney test $)$ in these six eyes on day $28(21.5$ photons/ms, $95 \%$ CI $12.7-$ $42 \cdot 2)$ than in the 21 eyes with an uneventful recovery $(11 \cdot 2$ photons/ms, 95\% CI $9 \cdot 2-14 \cdot 1)$. Despite these differences in the early postoperative period there was no statistically significant difference in median aqueous flare at 3 months between the two groups of eyes $(p=0 \cdot 7)$.

\section{THE CONSENSUAL RESPONSE OF THE BAB IN THE FELLOW EYE}

There was no evidence of a universal consensual $B A B$ response in the fellow eye. However, five patients showed a consensual increase in aqueous flare in the fellow eye of more than $50 \%$ over their preoperative value. This occurred in one patient on day 2 , in two patients on day 7 , and in a further two patients on day 28 . None of these five patients had marked inflammation in their operative eye (defined as a flare value of more than 150 photons/ms on the first postoperative day). However, three of these five patients were in the group who had an increase in flare in the operative eye between the second and seventh postoperative day. A particularly marked consensual response was observed in one of these patients, a man aged 68 years who developed an increase in flare in his operative eye and a simultaneous marked increase in aqueous flare in his fellow eye (Fig 5). This eye was also pseudophakic having been operated on only 6 weeks previously (outside of this series). There was a median percentage increase in aqueous flare at 1 month of $18 \cdot 2 \%(95 \% \mathrm{CI}-4 \cdot 8 \%$ to $+135 \cdot 8 \%$ ) over the preoperative value in the fellow eyes of the six patients who had an increase in flare in the operative eye during the first postoperative week, compared with a median decrease in flare of $7 \cdot 5 \%$ (95\% CI $-16 \%$ to $+9.5 \%$ ) over the same period in patients with an uneventful recovery, this difference almost reaching statistical significance $(p=0.05$, MannWhitney test).

\section{Discussion}

This study defines the recovery of the BAB in a group of normal eyes after routine endocapsular surgery. Group data shows that aqueous flare and cells were greatest on the first postoperative day and then declined rapidly in the first week and more gradually thereafter (Figs 1 and 2). There was a large variation between patients in aqueous flare on the first postoperative day (median aqueous flare 79.6, 95\% CI 50.6-100.8,

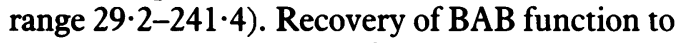
preoperative levels was found to occur by 3 months following surgery.

Factors such as intercurrent ocular or systemic disease, the surgeon, and type of surgery must influence postoperative recovery and the noninvasiveness and rapidity of laser photometry will allow a much more precise assessment of the effect that these have on the eye. There are, so far, only a few reports on the changes in aqueous flare and cells following cataract surgery. Tsuramiki et $a l^{8}$ found a similar peak in flare and cells on the first postoperative day with a decline thereafter but presented their results with a follow up of only 7 days. In another study of 48 eyes undergoing extracapsular cataract extraction and posterior chamber intraocular lens implantation, it was found that laser flare values in the operated eye remained elevated for 6 months when compared with a control group of 24 fellow eyes. ${ }^{9}$ Sato and Usui ${ }^{10}$ found that in four cases of juvenile cataract aqueous flare and cells had returned to their preoperative level in 4 months. Our results on the recovery of the BAB are consistent with the results of previous fluorophotometric studies: $69 \%$ of patients undergoing extracapsular cataract surgery and posterior chamber implantation had re-established the integrity of the BAB by 3 months. "This study used an age-matched group of normal eyes as their control group but most of the other studies have used the fellow unoperated eye as a control. In one such study of 63 eyes $^{12}$ recovery of the BAB had occurred by 3 months, whereas other authors ${ }^{13-15}$ have all reported that recovery of BAB function can continue for up to 6 months following surgery.

Despite the small numbers in this study we were able to demonstrate some variations in the inflammatory response within the first 7 days after surgery. In the majority of patients there was a consistent decline in both flare and cells from a peak on the first postoperative day (as shown in Figs 1 and 2). In a minority of patients (4/27 eyes) there was an asymptomatic increase in flare alone between the second and seventh postoperative days which resolved spontaneously (Fig 3) and in two patients there was a symptomatic increase in both flare and cells between day 2 and day 7 (Fig 4). It would appear that damage to the BAB occurs immediately after surgery but, in a small number of patients, is followed by an increase in inflammation within the first week and a delayed recovery of the BAB 
in the first postoperative month. Observations of other patients suggest this postoperative elevation of flare occurs as the lens capsule fibroses to the implant, possibly leading to damage of the anterior lens epithelial cells. These patterns of change in the BAB after cataract surgery need to be confirmed in larger studies but their time course correlates with the previously published reports of postoperative fibrinoid reactions. These reactions are, however, rare in our own practice.

In the previously quoted study of Tsurimaki et $a l^{8}$ the authors measured flare and cells daily in the first 7 postoperative days in a group of 49 eyes: $14(31 \%)$ of the eyes developed a fibrinous iritis. In these patients there was an initial decline in flare in the first 2 days and then an increase from day 3 onwards to reach a peak on day $5 . \mathrm{Nishi}^{16}$ found such a response in $7 \cdot 6 \%$ of eyes studied (45/596 eyes) and postulated that the production of collagen or its precursors as a result of lens epithelial cell fibrous pseudometaplasia produced a foreign body response in the anterior chamber with production of fibrin.

Although we were unable to detect the presence of a consensual response in the fellow eye of all patients following cataract surgery we were able to show its existence in some patients (Fig 5). Our results suggest that the occurrence of a consensual response is more common in eyes with a rise in flare postoperatively in the operative eye. Another study, ${ }^{17}$ using the laser flare cell meter, found no evidence of a consensual response in 20 patients in the first 3 months following cataract surgery. Miyake et al, ${ }^{6}$ using anterior segment fluorophotometry on eyes with posterior chamber lens implantation following phacoemulsification, found that a consensual reaction was a common occurrence, was highest on the first postoperative day, was proportional to the disruption of the $\mathrm{BAB}$ in the operative eye and comtinued during the first to fourth postoperative weeks. There are two possible mechanisms for a consensual response, firstly, the systemic release of a chemical mediator from an inflamed eye ${ }^{18}$ or, secondly, by neural transmission. ${ }^{19}$ In this study we found no relation between marked inflammation on the first postoperative day and the consensual response. The greatest consensual response in our series was seen in a patient with increasing flare in the operative eye during the first postoperative week and recent cataract surgery in the fellow eye (Fig 5). Larger studies are required to fully assess the role of previous intraocular surgery and type of inflammatory response in the development of a consensual response and the clinical relevance of these factors in some special situations such as in the timing of sequential surgery in patients with a damaged $\mathrm{BAB}$ from diabetes or uveitis.

This study was supported by charitable funds from The Iris Fund, The Star Foundation, and The Andrew Wilson Trust.

DJS is a member of the United States Scientific Advisory Committee to Kowa on the laser flare cell meter. The authors do not have any commercial or proprietary interest in any product, instrument, or company discussed in this manuscript. The statistical advice given by Mr Nicholas Taub, statistician, Department of Public Health Medicine, St Thomas's Hospital, London is ment of Public Health Med
gratefully acknowledged.

1 Sawa M, Tsurimaki Y, Tsuru T, Shimuzu H. New quantitative method to determine protein concentration and cell number in aqueous in vivo. $\mathcal{F}_{p} \mathcal{F}$ Ophthalmol 1988; 32: 13242

2 Shah SM, Spalton DJ, Smith SE. Measurement of aqueous cells and flare in normal eyes. Br $\mathcal{F}$ Ophthalmol 1991; 75: 348-52.

3 Oshika T, Nishi M, Mochizuki M, Nakamura M, Kawashima $\mathrm{H}$, Iwase $\mathrm{K}$, et al. Quantitative assessment of aqueous flare and cells in uveitis. $\mathscr{F}$ pn $\mathcal{F}$ Ophthalmol 1989; 33: 279-87.

4 Shah SM, Spalton DJ, Taylor JC. Correlations between laser flare cell measurements and anterior chamber protein con-

5 Spalton DJ, Shah SM. A comparison of the laser flare cell meter and anterior segment fluorophotometry in the assessment of damage to the blood-aqueous barrier following cataract surgery. ARVO Abstracts. Invest Ophthalmol Vis Sci 1991; 32 (suppl): 886.

6 Miyake K, Askura M, Maekubo K. Consensual reactions of human blood-aqueous barrier to implant operations. Arch Ophthalmol 1984; 102: 588-91.

7 Campbell MJ, Gardner MJ. Calculating confidence intervals for some non-parametric analyses. In: Gardner MJ, Altman DG, eds. Statistics with confidence. Confidence intervals and DG, eds. Statistics with confidence. Confidence intervals and
statistical guidelines. London: BMJ Publications, 1989: 71-3.

8 Tsurimaki Y, Sawa M, Shimuzu H. Pathogenetic analysis of post-operative protein concentration and cell count of fibrin exudate in the anterior chamber of the eye with a posterio chamber lens. Acta Soc Ophthalmol fpn 1988; 92: 1690-5.

9 Shan Z, Yong S, Sawa M, Masuda K. Quantitation of bloodaqueous barrier function after intraocular lens implantation. fpn f Clin Ophthalmol 1990; 44: 1997-2001.

10 Sato K, Usui M. Intraocular lens implantation in 5 children with cataract. $\mathcal{F}_{\text {pn }} \mathcal{f}$ Clin Ophthalmol 1991; 85: 802-5.

11 Ferguson VMG, Spalton DJ. Recovery of the blood aqueous barrier after cataract surgery. Br $\mathcal{F}$ Ophthalmol 1991; 75: 106-10.

12 Sanders DR, Kraff MC, Lieberman HL, Peyman GA, Tarabishy S. Breakdown and reestablishment of bloodaqueous barrier with implant surgery. Arch Ophthalmol 1982; 100: 588-90.

13 Kraff MC, Sanders DR, Peyman GA, Lieberman HL, Tarabishy S. Slit lamp fluorophotometry in intraocular lens patients. Ophthalmology 1980; 87: 877-80.

14 Majima Y. Consideration of the modern cataract surgery. Acta Soc Ophthalmol fpn 1982; 86: 1893-918.

15 Kaiya T. Observation of blood-aqueous barrier function after posterior chamber intraocular lens implantation. $\mathcal{F}$ Cataract Refract Surg 1990; 16: 320-4.

16 Nishi O. Fibrinous membrane formation on the posterior chamber lens during the early post-operative period. $\mathcal{f}$ Cataract Refract Surg 1988; 14: 73-7.

17 Katoh N, Komurasaki M, Miura M, Shinjo M, Nakagawa S, Iwake $M$, et al. Quantitation of consensual reaction following posterior chamber lens implantation. $f$ pn $\mathcal{f}$ Clin Ophthalmol 1989; 44: 1147-50.

18 Chiang TS, Thomas RP. Consensual ocular hypertensive response to prostaglandin. Invest Ophthalmol Vis Sci 1972; 11: $169-76$.

19 Perkins ES. Influences of the fifth cranial nerve on the intraocular pressure of the rabbit eye. Brf Ophthalmol 1957; 41: $257-300$. 